\title{
Peripheral Collisions of Relativistic Heavy Ions
}

\author{
C. A. Bertulani \\ Physics Department, Brookhaven National Laboratory, \\ Upton, New York 11973 *
}

October 31, 2018

\begin{abstract}
The physics of peripheral collisions with relativistic heavy ions (PCRHI) is reviewed. One- and two-photon processes are discussed.
\end{abstract}

PACS: 34.90.+q, 25.75.-q

\section{Introduction}

Peripheral collisions of relativistic heavy ions have attracted a great number of theoretical and experimental work (see, e.g., [1], and references therein). It is the purpose of this article to review these phenomena.

\section{Peripheral Collisions}

This field was born in 1924, when E. Fermi had an ingenious idea of relating the atomic processes induced by fast charged particles (the electron) to processes induced by electromagnetic waves. In 1934-1935, Weizsäcker and Williams corrected Fermi's calculation by including the appropriate relativistic corrections. The original Fermi's idea is now known as the WeizsäckerWilliams method [2], an approximation widely used in coherent processes

\footnotetext{
${ }^{*}$ Contribution to the Symposium on Fundamental Issues in Elementary Matter, In Honor and Memory of Michael Danos, Bad Honnef, Germany, September 2000.
} 
in QED and QCD. In this method the field generated by a fast particle is replaced by a flux of photons (QED), or a flux of by mesons and gluons (QCD) [3]. The number of equivalent photons, $n(\omega)$, of given energy, $\omega$, in peripheral collisions of relativistic heavy ions (PCRHI) can be calculated classically, or quantum-mechanically. For the electric dipole (E1) multipolarity both results are identical under the assumption of very forward scattering [3]. In ref. [3] the number of equivalent photons for all multipolarities was calculated exactly. It was shown that for the electric dipole multipolarity, E1, the equivalent photon number, $n_{E 1}(\omega)$, coincides with the one deduced by Weizsäcker and Williams. It was also shown that in the extreme relativistic collisions the equivalent photon numbers for all multipolarities agree, i.e, $n_{E 1}(\omega) \sim n_{E 2}(\omega) \sim n_{M 1}(\omega) \sim \ldots$ The cross sections for one- and two-photon processes depicted in figure $1(\mathrm{a}, \mathrm{b})$ are given by

$$
\sigma_{X}=\int d \omega \frac{n(\omega)}{\omega} \sigma_{X}^{\gamma}(\omega), \text { and } \sigma_{X}=\int d \omega_{1} d \omega_{2} \frac{n\left(\omega_{1}\right)}{\omega_{1}} \frac{n\left(\omega_{2}\right)}{\omega_{2}} \sigma_{X}^{\gamma \gamma}\left(\omega_{1}, \omega_{2}\right)
$$

where $\sigma_{X}^{\gamma}(\omega)$ is the photon-induced cross section for the energy $\omega$, and $\sigma_{X}^{\gamma \gamma}\left(\omega_{1}, \omega_{2}\right)$ is the two-photon cross section. Note that we do not refer to the photon momenta. The virtual photons are real: $q^{2}=0$, a relation always valid for PCRHI.

Most applications of PCRHI were reviewed in ref. [4]. Since then a great amount of work has been performed in this field. I will only be able to quote a short number of references.

\subsection{Bremsstrahlung and Delbrück scattering}

Bremsstrahlung (fig. 1c) is a minor effect in PCRHI «1 The cross section is proportional to the inverse of the square mass of the ions. Most photons have very low energies (infrared). For $10 \mathrm{MeV}$ photons the central collisions (CCRHI) deliver $10^{6}$ more photons than the PCRHI [5]. However, Bremsstrahlung could be relevant to obtain information on the elastic scattering of photons off unstable particles, like pions: $Z+\pi \longrightarrow Z+\pi+\gamma$. For a collider the Bremsstrahlung cross section is given by

$$
\frac{d \sigma_{\gamma}}{d \omega}=\frac{16 Z^{6} \alpha^{3}}{3 \omega A^{2} m_{N}^{2}} \ln \left(\frac{\gamma}{\omega R}\right)
$$


where $m_{N}$ is the nucleon mass, $\gamma=2 \gamma_{c}^{2}-1$, where $\gamma_{c}$ is the collider Lorentz gamma factor $\left(\gamma_{c} \sim 100\right.$ for RHIC/BNL), and $R$ is the nuclear dimension $\left(R \sim 2.4 A^{1 / 3} \mathrm{fm}\right)$ [5].

(a)
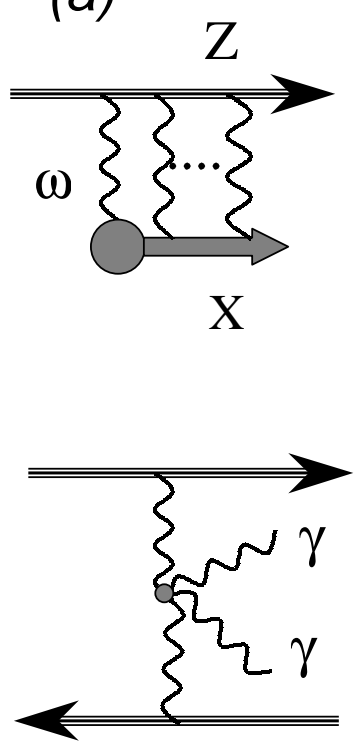

(d) (b)
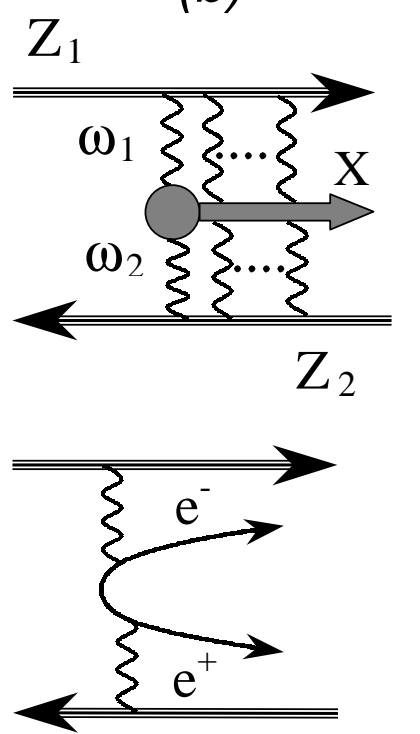

(e) (c)
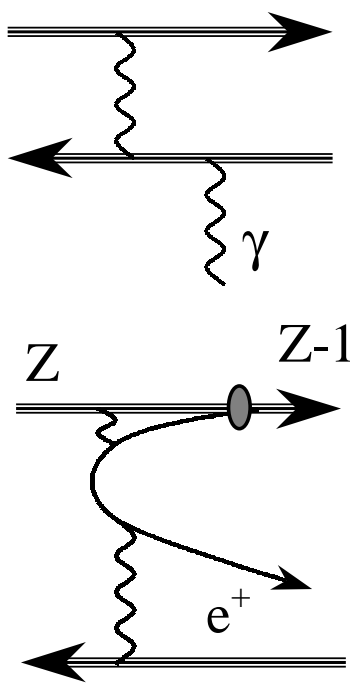

(f)

Figure 1: PCRHI processes: (a) one-photon, (b) two-photon, (c) Bremsstrahlung, (d) Delbrück scattering, (e) pair-production, and (f) pairproduction with capture.

Delbruck scattering $\left(\gamma^{*}+\gamma^{*} \longrightarrow \gamma+\gamma\right)$ involves an aditional $\alpha^{2}$ as compared to pair production and has never been possible to study experimentally. The cross section is about $50 \mathrm{~b}$ for the LHC [5] and the process is dominated by high-energy photons, $E_{\gamma} \gg m_{e}$. A study of this process in PCRHI is thus promising if the severe background problems arising from CCRHI can be eliminated. To my knowledge, no experiments of Bremsstrahlung or Delbrück scattering in PCRHI have been performed so far. The total cross 
section for Delbrück scattering $\left(\omega \gg m_{e}\right)$ in colliders is given by

$$
\sigma_{\gamma \gamma}=2.54 Z^{4} \alpha^{4} r_{e}^{2} \ln ^{3}\left(\frac{\gamma}{m_{e} R}\right)
$$

where $r_{e}=e^{2} / m_{e}$ is the classical electron radius [5].

\section{$2.2 \quad$ Atomic ionization}

Atomic ionization by RHI is used in experiments with fixed targets for the basic understanding of atomic structure physics in high-Z few electron atoms such as hydrogen-like or helium-like uranium atoms. A nice book on this subject has been written by Eichler and Meyerhof (see also the review by Anholt and Gould) 何.

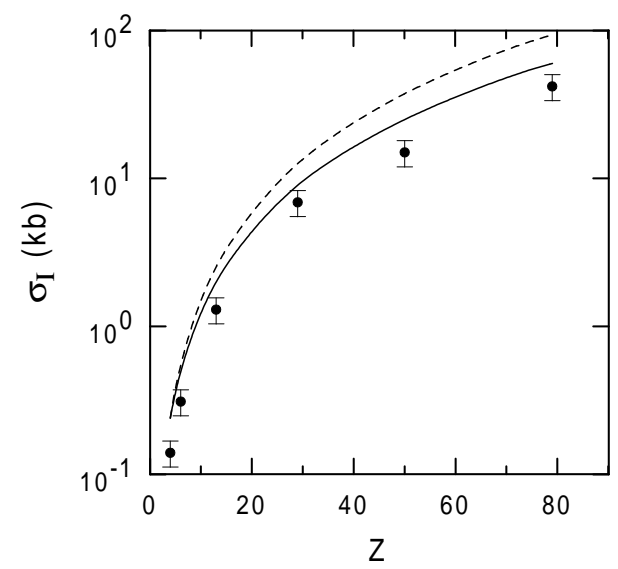

Figure 2: Atomic ionization cross sections for $\mathrm{Pb}^{81+}(33 \mathrm{TeV})$ beams on several targets [7].

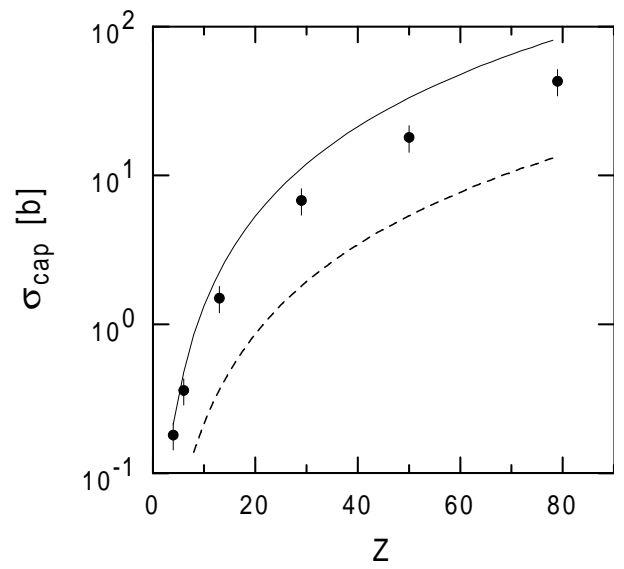

Figure 3: Pair production with capture for $\mathrm{Pb}^{82+}(33 \mathrm{TeV})$ beams on several targets [7].

The cross sections are very large, of order of kilobarns, increasing slowly with the logarithm of the RHI energy. For a fixed target experiment using bare naked projectiles one gets [4]:

$$
\sigma_{I}=\frac{Z_{P}^{2} r_{e}^{2}}{Z_{T}^{2} \alpha^{2}}\left[1.8 \pi+9.8 \ln \left(\frac{2 \gamma}{Z_{T} \alpha}\right)\right],
$$


which decreases with the target charge $Z_{T}$. This is due to the increase of the binding energy of $K$-electrons with the atomic charge. The first term is due to close collisions assuming elastic scattering of the electron off the projectile, while the second part is for distant collisions, with impact parameter larger than the Bohr radius. The probability to eject a $K$-electron is much larger than for other atomic orbitals. Recent experiments have reported ionization cross sections for $\mathrm{Pb}^{81+}(33 \mathrm{TeV})$ beams on several targets [7]. In this case, the role of projectile and target are exchanged in the previous equation. In figure 2 we show the results this equation (dashed line) compared to the experimental data. Since the targets are screened by their electrons, the discrepancy is expected. Even the most detailed calculations by Anholt and Becker [8] (solid line) yield larger cross sections than the experimental data.

Non-perturbative calculations, solving the time dependent Dirac equation exactly, were first performed by Giessen and Oak Ridge groups [10, 11]. It is claimed in ref. [7] that such non-perturbative calculations do in fact reproduce their data since they yield cross sections which are approximately $70 \%$ the perturbative ones 90. However, there are little data available for a decisive conclusion about an appropriate theory.

\subsection{Free and bound-free electron-positron pair pro- duction}

Between 1933 and 1937, Furry, Carlson, Landau, Lifshitz, Bhabha, Racah, Nishina, Tomonaga, and several others performed calculations of $e^{+} e^{-}$production in relativistic collisions of fast particles (cosmic rays) [12]. The purpose was to test the newly born Dirac theory for the positron. Starting with the Dirac equation for the electron and its antiparticle they obtained that, to leading order in $\gamma$,

$$
\sigma=\frac{28}{27 \pi} Z_{P}^{2} Z_{T}^{2} r_{e}^{2} \ln ^{3}\left(\frac{\gamma}{4}\right)
$$

Unfortunately, in view of the experimental difficulties, these results could never been fully tested. A renewed interest in this process appeared with the construction of relativistic heavy ion accelerators. For heavy ions with very large charge (e.g, lead, or uranium) the pair production probabilities and cross sections are very large. They cannot be treated to first order in perturbation theory [4], and are difficult to calculate. This resulted in a great 
amount of theoretical studies [13]. The formulation of the problem with use of numerical algorithms has varied wildly among several groups. Semianalytical approaches have also been used. The comparison among all these results is rather deceiving, since very different results are obtained for the production cross sections, sometimes differing by orders of magnitude. The perturbative calculations are simple to write down, but involve rather complicated integrals, specially for low energy electrons, due to the distortion and relativistic effects on the continuum electronic wavefunction [6]. Screening is also a source of problems. The non-perturbative calculations are simpler to formulate, but are useless without a numerical algorithm which contains implicit approximations.

Recently, good developments in more tractable formulations of the problem appeared in the literature. One replaces the Lorentz compressed electromagnetic fields by delta functions, works with light cone variables, and obtains almost closed form expressions [14]. However, some of these works have been strongly criticized [15] because they do not account properly for Coulomb distortion of the lepton wavefunctions. This problem was addressed again in ref. [16] with the conclusion that a full account of distortion of the leptonic wavefunctions has not been achieved so far. In other words, no theory has ever been possible to tackle the multiple photon exchange between the electron (or positron) with both the target and the projectile. A simple thought reveals why the Coulomb distortion is so important. In the frame of reference of one of the nuclei, the energy spectrum of the emitted electron (or positron) is peaked at $\varepsilon \sim 2 m_{e}$ [4]. This peak is due to the Coulomb attraction (repulsion) which eliminates low energy components of the leptonic wavefunctions, combined with the decrease in energy of the number of equivalent photons generated by the other nucleus. Changing frame of reference, changes this picture. Thus, a correct calculation should yield two peaks in the energy distribution of the electron (or positron): one at $\varepsilon \sim 2 m_{e}$, and another around $\varepsilon \sim 2 \gamma_{c}^{2} m_{e}$. The reason it does not appear in perturbative calculations is that the distortion on the leptonic wavefunctions are calculated with respect to only one of the nuclei. Accounting for the distortion with respect to both nuclei, suggests that the total cross section should be about twice the value obtained by Landau and others [12]. This seems to be a challenge for present theoretical calculations.

An important phenomenon occurs when the electron is captured in an atomic orbit of the projectile, or of the target. In a collider this leads to 
beam losses each time a charge modified nucleus passes by a magnet downstream [5]. A striking application of this process was the recent production of antihydrogen atoms using relativistic antiproton beams [17]. Here the positron is produced and captured in an orbit of the antiproton. Early cal-

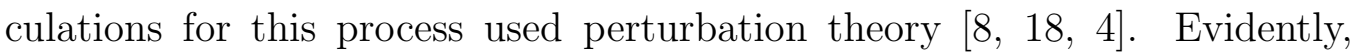
the best way to perform the calculation is using the frame of reference of the nucleus where the electron is captured. Many other calculations have been performed [13]. Some of them used non-perturbative approaches, e.g., coupled-channels calculations. Initially some discrepancy with perturbative calculations were found, but later it was shown that non-perturbative calculations agree with the perturbative ones at the $1 \%$ level (see, e.g., first reference of [14]). In fact, it would be a surprise if a different result was found. The first term of the perturbation series is already small enough to neglect the inclusion of higher order terms [四].

The expression

$$
\sigma=\frac{3.3 \pi Z^{8} \alpha^{6} r_{e}^{2}}{\exp (2 \pi Z \alpha)-1}\left[\ln \left(0.681 \gamma_{c}^{2}\right)-\frac{5}{3}\right]
$$

for pair production with electron capture in PCRHI was obtained in ref. [四]. The term $[\ldots]^{-1}$ is the main effect of the distortion of the positron wavefunction. It arises through the normalization of the continuum wavefunctions which accounts for the reduction of the magnitude of the positron wavefunction near the nucleus where the electron is localized (bound). Thus, the greater the $Z$, the less these wavefunctions overlap. The above equation also predicts a dependence of the cross section in the form

$$
\sigma=A \ln \gamma_{c}+B
$$

where $A$ and $B$ are coefficients depending on the system. This dependence was used in the analysis of the experiment in ref. [7]. In recent calculations, attention was given to the correct treatment of the distortion effects in the positron wavefunction [20]. In figure 3 we show the recent experimental data of ref. [7] compared to the above equation and recent calculations (second reference of [20]). These calculations also predict a $\ln \gamma_{c}$ dependence but give larger cross sections than in ref. [4]. The comparison with the experimental data is not fair since atomic screening was not taken into account. When screening is present the cross sections will always be smaller by at least a 
factor 2-4 [4]. The conclusion here is that pair production with electron capture is a process which is well treated in first order perturbation theory. The main concern is the correct treatment of distortion effects (multiphoton scattering) 20.

\subsection{Relativistic Coulomb excitation and fragmentation}

Relativistic Coulomb excitation is becoming a popular tool for the investigation of the intrinsic nuclear dynamics and structure of the colliding nuclei [21]. This is specially important in reactions involving radioactive nuclear beams [22]. Coulomb excitation and dissociation of such nuclei are common experiments in this field [1, 23]. The advantage is that the Coulomb interaction is very well known. The real situation is more complicated since the contribution of the nuclear-induced processes cannot be entirely separated in the experimental data. The treatment of the dissociation problem by nuclear forces is very model dependent, based on eikonal or multiple Glauber scattering approaches [22, 24]. Among the uncertainties are the in-medium nucleon-nucleon cross sections at high-energies, the truncation of the multiple scattering process and the separation of stripping from elastic dissociation of the nuclei [25]. Nonetheless, specially for the very weakly-bound nuclei, relativistic Coulomb excitation has lead to very exciting new results [22, 24].

The Coulomb breakup of ${ }^{11} \mathrm{Li}$ has lead to interesting results which gave rise to a series of speculations about the reaction mechanism, the dynamics, and the structure of this nucleus. One speculates if the reaction proceeds under a single or multiple photon-exchange between the projectile and the target. In the first case, perturbation theory gives a straight relation between the data and the matrix element for electromagnetic dissociation. Such matrix elements are the clearest probes one can get about the nuclear structure of these nuclei. In the second case, often called by post-acceleration effects [24], one has to perform a non-perturbative treatment of the reaction what complicates the extraction of the electromagnetic (mainly E1) matrix elements. By solving such problems one expects to learn if the Coulombinduced breakup proceeds via a resonance or by the direct dissociation into continuum states 24. There is a strong ongoing effort to use the relativistic Coulomb excitation technique also for studying the excitation of bound excited states in exotic nuclei, to obtain information on gamma-decay widths, angular momentum, parity, and other properties of hitherto unknown states 


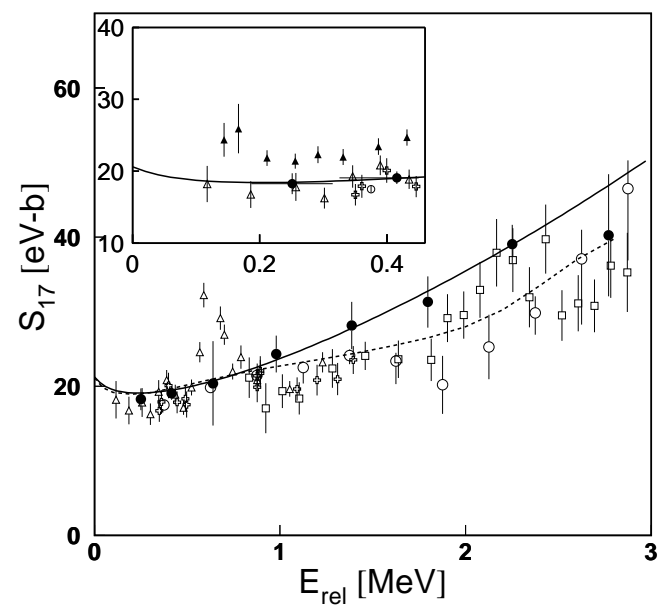

Figure 4: S-factors for the ${ }^{7} B e(p, \gamma)^{8} B$ reaction.

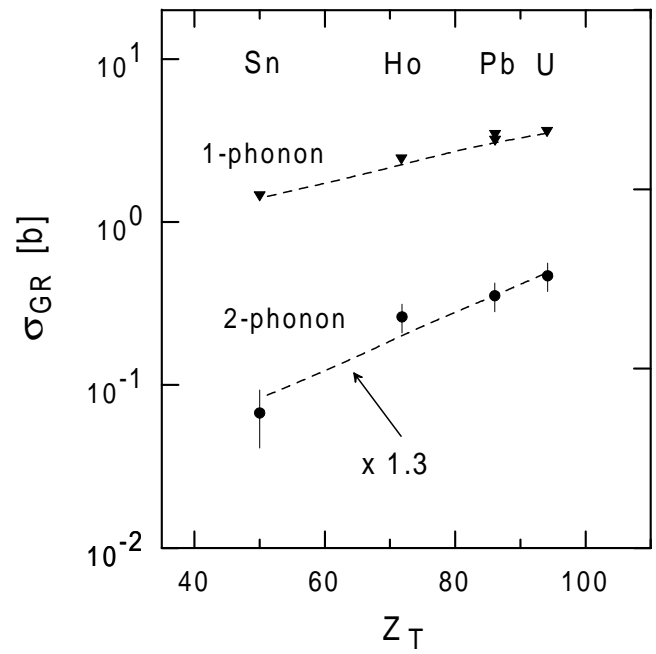

Figure 5: Cross sections for the excitation of the GDR and the DGDR.

[23].

Another application of Coulomb dissociation of radioactive nuclei is in astrophysics. Radiative capture reactions are known to play a major role in astrophysical sites, e.g., in a pre-supernova [26]. Some of these reactions, like for example, ${ }^{7} B e(p, \gamma){ }^{8} B$, can be studied via the inverse photo-dissociation reaction ${ }^{8} B(\gamma, p)^{7} B e$. One often uses the astrophysical S-factor, defined by

$$
S(E)=E \sigma(E) \exp [-2 \pi \eta(E)], \quad \text { where } \quad \eta(E)=\frac{Z_{1} Z_{2} e^{2}}{\hbar \sqrt{2 \mu_{12} E}},
$$

where $E$ is the relative kinetic energy of the two nuclei. The matrix elements involved in the dissociation processes are the same as those involved in the absorption by real photons [1]. One of the experiments using this technique was performed at the GSI/Darmstadt [27]. The S-factor obtained in this experiment is shown in figure 4 as solid circles. Such experiments are very important specially in those cases where the radiative capture cross section is so small that the direct fusion experiments are very difficult, or even impossible to carry out. The contribution of the nuclear-induced breakup and of post-acceleration effects also limit the use of the Coulomb dissociation tech- 
nique for this purpose. But, in view of the much more difficult experiments on direct capture, it is a very useful alternative method.

Another important application of relativistic Coulomb excitation is the study of the DGDR (Double Giant Dipole Resonance). A GDR occurs in nuclei at energies of 10-20 MeV. Assuming that these are harmonic vibrations of protons against neutrons, one expects that DGDRs, i.e., two giant dipole vibrations superimposed in one nucleus, will have exactly twice the energy of the GDR 四, 21. Small deviations are expected from non-harmonic properties of the nuclear response. A series of experiments at the GSI/Darmstadt have obtained energy spectra, cross sections, and angular distribution of fragments following the decay of the DGDR. Initially they observed cross sections twice as large as expected from theoretical calculations. These results lead to a series of studies on deviations from the harmonic picture of the giant resonances. More recently, new experiments and new analysis have shown that the experimental cross sections are only about $30 \%$, or less, bigger than the theoretical ones. This is shown in figure 5 where the cross sections for the excitation of 1-phonon (GDR),

$$
\sigma_{1} \sim 2 \pi S \ln \left[\frac{2 \gamma A_{T}^{1 / 3}}{A_{P}^{1 / 3}+A_{T}^{1 / 3}}\right]
$$

while for the 2-phonon state it is

$$
\sigma \sim \frac{S^{2}}{\left(A_{P}^{1 / 3}+A_{T}^{1 / 3}\right)^{2}}, \quad \text { where } \quad S=5.45 \times 10^{-4} \frac{Z_{P}^{2} Z_{T} N_{T}}{A_{T}^{2 / 3}} \mathrm{mb}
$$

The dashed lines of figure 5 are the result of more elaborate calculations [21]. The GSI experiments are very promising for the studies of the nuclear response in very collective states. One should notice that after many years of study of the GDRs and other collective modes, the width of these states are still poorly explained theoretically, even with the best microscopic approaches known sofar. The extension of these approaches to the study of the width of the DGDRs will be helpful to improve such models. Hopefully, after addressing more fundamental questions on QCD and QED of strong electromagnetic fields, some experiments on relativistic colliders will also be proposed for the study of nuclear structure issues, specially the issue of the DGDR. 
The DGDR contributes only to about $10 \%$ of the total fragmentation cross section induced by Coulomb excitation with relativistic heavy ions. The main contribution arises from the excitation of a single GDR, which decays mostly by neutron emission. This is also a potential source of beam loss in relativistic heavy ion colliders [1], and an important fragmentation mode of relativistic nuclei in cosmic rays.

\subsection{Meson and hadron production}

The production of heavy lepton pairs $\left(\mu^{+} \mu^{-}\right.$, or $\left.\tau^{+} \tau^{-}\right)$, or of meson pairs (e.g., $\pi^{+} \pi^{-}$) can be calculated using the second of equation (1). One just needs the cross sections for $\gamma \gamma$ production of these pairs. Since they depend on the inverse of the square of the particle mass [5], the pair-production cross sections are much smaller in this case. The same applies to single meson production by $\gamma \gamma$ fusion. The $\gamma \gamma$ cross section is given by

$$
\sigma_{\gamma \gamma \rightarrow M}=8 \pi^{2}(2 J+1) \frac{\Gamma_{M \rightarrow \gamma \gamma}}{M} \delta\left(W^{2}-M^{2}\right),
$$

where $J, M$, and $\Gamma_{M \rightarrow \gamma \gamma}$ are the spin, mass and two-photon decay width of the meson, $W$ is the c.m. energy of the colliding photons [5]. A correction for the equivalent photon numbers is necessary, since the two-photon energy folding in eq. (1) has to account for the space geometry of the two-photon collision [28].

A careful study of the production of meson pairs and single mesons in PCRHI was performed recently in ref. [29]. In table I we show the magnitude of the cross sections for single meson production at RHIC and at LHC [29]. Also shown are the cross sections due to difractive processes (pomeronpomeron exchange). We see that they are several orders of magnitude smaller than those from $\gamma \gamma$ fusion. The cross sections for the production of $\eta_{c}, \eta_{c}^{\prime}$ and $\eta_{b}$ are very small due to their higher masses. Similar studies have been done for meson production in $\gamma$-nucleus interactions. Particles like $\Delta, \rho, \omega$, $\phi, J / \Psi$, etc, can be produced in this way [30].

The possibility to produce a Higgs boson via $\gamma \gamma$ fusion was suggested in ref. [31]. The cross sections for LHC are of order of 1 nanobarn, about the same as for gluon-gluon fusion. But, the two-photon processes can also produce $b \bar{b}$ pairs which create a large background for detecting the Higgs boson. A good review of these topics was presented in ref. [30]. 
Table 1: Particle production in PCRHI at RHIC and at LHC. Masses are in $\mathrm{MeV}$, decay widths in $\mathrm{keV}$, and cross sections in mb. The cross sections are for $\gamma \gamma$ and pomeron-pomeron $(\mathcal{P} \mathcal{P})$ exchange processes, respectively.

\begin{tabular}{lllllll}
\hline Meson & $\mathrm{M}$ & $\Gamma_{X \rightarrow \gamma \gamma}$ & $R H I C_{\gamma \gamma}$ & $L H C_{\gamma \gamma}$ & $R H I C_{\mathcal{P P}}$ & $L H C_{\mathcal{P P}}$ \\
\hline$\pi^{0}$ & 135 & $8 \times 10^{-3}$ & 7.1 & 40 & 0.05 & 0.367 \\
$\eta$ & 547 & 0.463 & 1.5 & 17 & 0.038 & 0.355 \\
$\eta^{\prime}$ & 958 & 4.3 & 1.1 & 22 & 0.04 & 0.405 \\
\hline
\end{tabular}

\section{Acknowledgment(s)}

The author is currently a fellow of the John Simon Guggenheim Memorial Foundation. This work was partially supported by the Brazilian funding agencies CNPq, CAPES, FUJB and MCT/FINEP/CNPQ(PRONEX) under contract No. 41.96.0886.

\section{References}

[1] C.A. Bertulani and G. Baur, Physics Today, March 1994, p. 22.

[2] E. Fermi, Z. Physik 29, 315 (1924); K. F. Weizsacker, Z. PhysikE, 612 (1934); E. J. Williams, Phys. Rev. 45, 729 (1934).

[3] C.A.Bertulani and G. Baur, Nucl. Phys. A442 (1985) 73.

[4] C.A. Bertulani and G. Baur, Physics Reports 163 (1988) 299.

[5] G. Baur and C.A. Bertulani, Z. Phys. A330 (1988) 77; C.A. Bertulani and G. Baur, Nucl. Phys. A505 (1989) 835.

[6] J. Eichler and. Meyerhof, Relativistic Atomic Collisions (Academic Press, San Diego, 1995); R. Anholt and H. Gould, in Advances in Atomic and Molecular Physics (Academic Press, San Diego, 1986), p. 315.

[7] H.F. Krause et al., Phys. Rev. Lett. 80 (1998) 1190.

[8] R. Anholt and U. Becker, Phys. Rev. A36 (1987) 4628. 
[9] A.J. Baltz, M.J. Rhoades-Brown and J. Weneser, Phys. Rev. A44 (1991) 5668; 47 1993) 344; 48 (1993) 2002; 50 (1994) 4842

[10] U. Becker, N. Grün and W. Scheid, J. Phys. B16 (1983) 1967; U. Becker, PhD. Thesis, Giessen (1986).

[11] C. Bottcher and M.R. Strayer, Phys. Rev. Lett. 54 (1985) 669.

[12] W.H. Furry and J.F. Carlson, Phys. Rev. 44 (1933) 238; L.D. Landau and E.M. Lifshitz, Phys. Zs. Sowjet, 6 (1934) 244; H.J. Bhabha, Proc. R. Soc. London Ser. A152 (1935) 559; Y. Nishina, S. Tomonaga and M. Kobayashi , Sci. Pap. Phys. Chem. Res. 27 (1935) 137.

[13] C. Bottcher and M.R. Strayer, Phys. Rev. D39 (1989)1330; J. Phys. G16 (1990) 975; Phys. Lett. B237 (1990) 175; G. Baur, Phys. Rev. D41 (1990) 3535; Phys. Rev. A42 (1990) 5736; M.J. Rhoades-Brown and J. Weneser, Phys. Rev. A4 (1991) 33; C. Best, W. Greiner and G. Soff, Phys. Rev. A46 (1992) 261; M. Vidović, M. Greiner, C. Best and G. Soff, Phys. Rev. C47 (1993) 2308; K. Hencken, D. Trautmann and G. Baur, Phys. Rev. A51 (1995) 998; A51 (1995) 1874;

[14] A.J. Baltz, Phys. Rev. Lett. 78 (1997) 1231; A.J. Baltz and L. McLerran, Phys. Rev. C58 (1998) 1679; B. Segev and J.C. Wells, Phys. Rev. A57 (1998) 1849; U. Eichmann, J. Reinhardt, S. Schramm and W. Greiner, Phys. Rev. A59 (1999) 1223.

[15] D.Yu. Ivanov, A. Schiller and V.G. Serbo, Phys. Lett. B454 (1999) 15; R.N. Lee and A.I. Milstein, e-print hep-ph/9909452.

[16] J. Reinhardt, S. Schramm and W. Greiner, Phys. Rev. A61, 062710 (2000); Phys. Rev. C61, 064901 (2000)

[17] G. Baur et al., Phys. Lett. B368 (1996) 351; G. Blanford et al., Phys. Rev. Lett. 80 (1998) 3037.

[18] U. Becker, J. Phys. B20 (1987) 6563.

[19] K. Momberger, N. Grün and W. Scheid, Z. Phys. D18 1991) 133; K. Rumrich, G. Soff and W. Greiner, Phys. Rev. A47 1993) 215; K. Momberger, A. Belkacem and A.H. Sorensen, Europhys. Lett. 32 (1995) 401. 
[20] H. Meier et al., e-print nucl-th/0008020; C.A. Bertulani and D. Dolci, e-print nucl-th/0008015.

[21] T. Aumann, P.F. Bortignon, H. Emling, Ann. Rev. Nucl. Part. Sci. 48 (1998) 282; C.A. Bertulani and V.Yu. Ponomarev, Phys. Reports 321 (1999) 139.

[22] C.A. Bertulani, L.F. Canto and M.S. Hussein, Phys. Rep. 226 (1993) 282; T. Glasmacher, Ann. Rev. Nucl. Part. Sci. 48 (1998) 1.

[23] S.A. Austin and G.F. Bertsch, Scientific American 272 (1995) 62; P. G. Hansen, A. S. Jensen, and B. Jonson, Ann. Rev. Nucl. Part. Sci. 45, 591 (1995).

[24] G. Baur, C.A. Bertulani and D.M. Kalassa, Nucl. Phys. A550 (1992) 527; K. Ieki, et al., Phys. Rev. Lett. 70 (1993) 730; G.F. Bertsch and C.A. Bertulani, Nucl. Phys. A556 (1993) 136; Phys. Rev. C49 (1994) 2834; Nucl. Phys. A581 (1995) 107

[25] M.S.Hussein, R.A.Rego and C.A. Bertulani, Phys. Reports 201 (1991) 279; C.A. Bertulani, P. Lotti and H. Sagawa, Phys. Rev. C57 (1998) 217

[26] D.D. Clayton, Principles of Stellar Evolution and Nucleosynthesis, McGraw-Hill, New York, 1968; C. Rolfs and W.S. Rodney, Cauldrons in the Cosmos, Chicago Press, Chicago, 1988

[27] N. Iwasa, F. Boué, G. Surówka, et al., Phys. Rev. Lett. 83 (1999) 2910

[28] G. Baur and L.G. Ferreira Filho, Nucl. Phys. A518 (1990) 786; N. Cahn and J.D. Jackson, Phys. Rev. D42 (1990) 3690

[29] C.G. Roldão and A.A. Natale, e-print nucl-th/0003038; K.A. Chikin et al., e-print nucl-th/0002028

[30] G. Baur, K. Hencken and D. Trautmann, J. Phys. G24 (1998) 1657

[31] M. Grabiak, B. Müller, W. Greiner and P. Koch, J. Phys. G15 (1989) L25; E. Papageorgiu, Phys. Rev. D40 (1989) 92 